\title{
Warum der Patient kein Kunde ist
}

Christian K. Schweiger

Korrespondenz:

Christian K. Schweiger

Arzt

Unterer Michlenberg 10

CH-9038 Rehetobel

sittahbeing@gmail.com
Der Kunde soll ja bekanntlich König sein und muss demnach auch entsprechend seinem Rang im Umfeld von Angebot und Nachfrage geradezu königlich umworben werden. Seit einiger Zeit wird nun, aus noch näher zu belegenden Gründen, auch in der Medizin den Gesetzmässigkeiten der Marktwirtschaft eine zunehmende Bedeutung beigemessen.

Dabei war das primum mobile der Initianten eines derartigen Denkprozesses nicht etwa das Wohl des Patienten, sondern standen dabei solch profane Kriterien wie Kostendeckung und Ressourcenknappheit im Medizinwesen im Vordergrund. Der Patient musste also neu definiert werden, quasi in seiner Funktion und Bedeutung neu verstanden sein!

Hierzu musste dem Kinde zunächst ein neuer Name gegeben werden, der einerseits im marktwirtschaftlichen Vokabular plausibel wäre, andererseits aber nicht mit der Aura von Krankheit und Leid in Verbindung gebracht würde.

Der Patient als Kunde war geboren.

Im Denken vordergründig progressiver Mediziner und deren Verwaltung soll nun dem Kunden natürlich nur allererste Qualität offeriert werden, also die medizinische Leistung ähnlich einem «Haute-Couture-Objekt» vermarktet werden. Dabei soll die Qualität durch sogenannte TQM-Kurse (Total Quality Management) sichergestellt werden. Ganze Spitäler werden dann in der Folge ISO-zertifiziert, gleichsam mit einem Qualitätslabel versehen, wie wir es aus dem gewinnorientierten produzierenden Gewerbe seit langem kennen und dessen Wurzeln in der japanischen Fliessbandindustrie der sechziger Jahre liegen. Der Kunde im Patienten soll dann also bereits durch den Qualitätsstempel des Spitals Vertrauen fassen und sich dem Angebot entsprechend eine für ihn massgeschneiderte Variante, eventuell im Sinne einer Operation, aussuchen, besser einkaufen. Unschwer werden wir demzufolge die Begriffspaare Angebot und Nachfrage sowie Arzt und Krankheit korrelieren.

Die ärztliche Leistung wird die Angebotsseite repräsentieren, die durch geschicktes Marketing also den durch Krankheit in den Rang eines Nachfragers erkorenen ehemaligen Patienten, für sich gewinnen will.

Sofort mag sich der geneigte Leser fragen, ob denn nun die Nachfrage das Angebot oder das Angebot die Nachfrage regelt? Natürlich kann

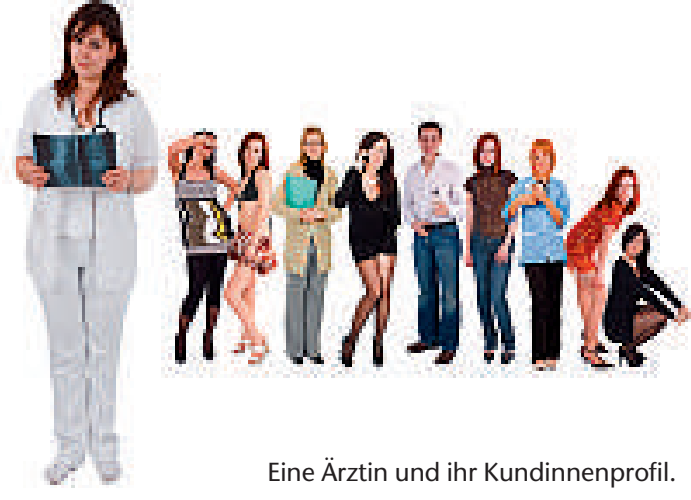

die Wirtschaft durch geeignetes Instrumentarium wie Werbung und im weitesten Sinne Konsumanimation einen künstlichen Bedarf und somit Nachfrage anregen, kaum wird es jedoch noch so findigen Strategen auf ärztlicher Seite gelingen, den Menschen Krankheit als etwas Erstrebenswertes zu suggerieren, um sich damit potentielle Nachfrager, also Patienten, zu generieren.

Dabei wird einerseits von der Gesellschaft «Fliessbandmedizin» schon seit langem kritisiert, nun aber wird andererseits das Vokabular des Marktes anscheinend widerspruchslos akzeptiert und damit zukünftig, nachdem die neue Sprache gleichermassen von allen erlernt wurde, die Befindlichkeit des Menschen zum variablen Nachfragefaktor des Homo oeconomicus degeneriert, wo Physis und Psyche des Patienten zum austauschbaren Handelsgut eines Kunden mutieren.

Beispiele eines derartigen Szenarios lassen sich bereits erkennen, wenn etwa «The Swiss Leading Hospitals» in Anspielung auf ihr Pendant, die Luxushotellerie, um «Gäste» werben.

«Aufhebung des Kontrahierungszwanges Members only» könnte es darüber hinaus künftig dem Patienten entgegenschallen, wenn er wegen einer Unpässlichkeit einen Arzt aufsuchen möchte und dessen «Krankenkassenkontingent» gerade nicht mit der jeweiligen Krankenversicherung des Patienten kompatibel ist, er quasi die falsche Kreditkarte besitzt.

Somit wird schon alleine die fatalistische Komponente der Krankheit das Wechselspiel von Angebot und Nachfrage in der Medizin nicht funktionieren lassen.

Schon zeigt sich, dass die Instrumente und Gesetze der Marktwirtschaft, sogar wenn diese noch sozial zu nennen wäre, nicht auf die Realitäten des Medizinwesens zu übertragen sind. 
Der Patient wird eben nicht aus freien Stücken ein Spital aufsuchen und sich zu einem, wenn auch noch so geschickt vermarkteten, chirurgischen Eingriff animieren lassen, wenn es aus gesundheitlichen Erwägungen heraus nicht unbedingt sein muss. Die Komponenten der Freiwilligkeit und des Hedonismus scheiden also aus, so wie diese andererseits als bestimmende Elemente in der Marktwirtschaft eindeutig ihren Stellenwert haben.

Des weiteren wird die medizinische Leistung ja durch ein Versicherungssystem finanziert, welches den Prinzipien der Solidarität und Subsidiarität gehorchen sollte, also Grundsätzen, die dem Wirtschaftskapitalismus eher fremd sein dürften.

Der Patient wäre also im neuen Sprachgebrauch ein Kunde, der eine Leistung in Anspruch nimmt, deren Bezahlung er aus einem Kollektivvermögen zu tätigen gedenkt, zu dessen Entstehung er durch seine Beitragszahlungen verhältnismässig geringfügig beigetragen hat, wenn wir einmal den «lebenslang kerngesunden Konstitutionstyp» nicht berücksichtigen wollen.

Das Bestreben der nun als Anbieter deklarierten Mediziner ist es dabei, möglichst diejenigen Kunden/Patienten zu akquirieren, durch deren Obolus sie dem Kollektivvermögen der Versicherten möglichst viel entnehmen können. Die medizinische Leistung wird eben nicht als solche vom Patienten beglichen, der sich seinen Leistungserbringer im besten Fall im Vorhinein durch Qualitätsvergleiche aussuchen konnte, sondern wird der Gesamtzahl der Leistungserbringer aus dem zunehmend insolvenzgefährdeten Kollektivvermögen der Versicherten rückvergütet. Darüber hinaus wächst die Anspruchshaltung der Versicherten seit Jahren exponentiell, wobei den technischen Errungenschaften, wie z.B. in den bildgebenden Verfahren (MRI/CT), ein Vertrauen entgegengebracht wird, welches der tatsächlichen Aussagekraft dieser Techniken in deren inflationärer Anwendung keinesfalls gerecht wird. Ohne Zweifel ist der exorbitante Kostenanstieg im Gesundheitswesen der Auslöser entsprechender Sparmodelle und somit auch des Bestrebens, marktwirtschaftliche Regelmechanismen anzuwenden.

Wie vorgängig erläutert, sind nun aber eben genau diese Gesetze und Selbstverständlichkeiten der Marktwirtschaft auf diejenigen der Medizin, nur marginal übertragbar, so dass sich die Frage aufdrängt, weshalb dann das betriebswirtschaftliche Vokabular dennoch gelten soll.

Wenn eine Gesellschaft Krankheit zunehmend stigmatisiert, wird in der Zukunft der erkrankte Mensch sein temporäres Ausscheiden aus dem Arbeitsmarkt womöglich lediglich noch als ein «Konsumverhalten spezieller Prägung» begreifen wollen, und dementsprechend selbst noch im Krankenstand im System von Angebot und Nachfrage funktionieren wollen, gleichsam die momentane Unpässlichkeit als «Konsumsignal» interpretieren. So sind die Wurzeln der geschilderten Abstraktion des Patienten, durch die er aus seiner ehemals geschützten Nische vertrieben werden soll, also mit einiger Wahrscheinlichkeit im ubiquitären unreflektierten Leistungsdenken einer Egogesellschaft zu suchen, in deren Selbstverständnis die leistungsstarke Gesundheit des Individuums immer mehr zur conditio sine qua non wird.

Jedoch wird die Unvereinbarkeit von menschlichem Wohlbefinden und dessen aktuell modisch apostrophierter Materialisierung und Kommerzialisierung auch zukünftig den Patienten glücklicherweise nicht in den Adelsstand erheben können und eine altruistische Gegenströmung in der Medizin wird hoffentlich aufbegehren. 